\title{
Overcoming technical challenges when treating atypical hemolytic uremic syndrome with therapeutic plasma exchange
}

This article was published in the following Dove Press journal: International Journal of Nephrology and Renovascular Disease 18 November 2013

Number of times this article has been viewed

\section{Edward Zimbudzi}

Department of Nephrology, Monash Health, Monash Medical Centre, Victoria, Australia
Correspondence: Edward Zimbudzi Department of Nephrology, Monash Medical Centre, 246 Clayton Road, Clayton, Victoria 3168, Australia Tel +6I 395943618

Fax +6I 395946907 Email edward.zimbudzi@southernhealth. org.au

\begin{abstract}
Atypical hemolytic uremic syndrome (aHUS) is a very rare, life-threatening, progressive disease that frequently has a genetic component and in most cases is triggered by an uncontrolled activation of the complement system. Successful treatment of aHUS with plasma infusions and therapeutic plasma exchange (TPE) is well reported. TPE has been the treatment of choice in most adult patients with aHUS. However, due to severe hemolysis, which is common among aHUS patients, there are some technical challenges that can affect TPE treatment such as the continuous activation of the blood leak alarm due to hemolysis. Our experience shows that such patients can be managed better on a centrifuge based TPE machine compared to a membrane based TPE machine.
\end{abstract}

Keywords: atypical hemolytic uremic syndrome, aHUS, blood leak alarm, centrifuge based TPE, membrane based TPE, therapeutic plasma exchange, TPE

\section{Introduction}

Hemolytic uremic syndrome (HUS) is a hematological disease characterized mainly by the triad of microangiopathic hemolytic anemia, thrombocytopenia, and acute renal failure. It is commonly caused by infections of Shiga-like toxin producing bacteria, such as Escherichia coli strain O157:H7, O111:H8, O103:H2, O123, and O26. ${ }^{1}$ Diarrhea is often present in such cases which can also be referred to as typical HUS. On the other hand, atypical HUS (aHUS) is characterized by the absence of diarrheal illness and can be acquired, genetic, or idiopathic. aHUS represents approximately $10 \%$ of all HUS cases. ${ }^{2}$ Currently, plasma exchange and/or plasma infusions is the recommended first-line management for aHUS. ${ }^{3}$ This paper presents the technical challenges faced while treating a patient who had aHUS with therapeutic plasma exchange (TPE).

\section{Case report}

A 21-year-old female with a known history of aHUS presented to our emergency department in September 2012. She complained of general aches and pains consistent with her previous episodes of aHUS. She had three relapses before when she was aged 8, 10, and 20. They all occurred in September. She presented with 3 days of fever and sore throat followed by dark urine. On examination, she was found to be afebrile, alert, oriented, and she was talking in full sentences. Her sitting blood pressure was $140 / 90 \mathrm{mmHg}$ and her heart rate was 70 beats per minute with an oxygen saturation of $99 \%$ on room air.

Hematological investigations revealed a hemoglobin $(\mathrm{Hb})$ count of $96 \mathrm{~g} / \mathrm{L}$, platelet count $16 \times 10^{9} / \mathrm{L}$, red cell count $3.01 \times 10^{12} / \mathrm{L}$, and a hematocrit of $0.27 \mathrm{~L} / \mathrm{L}$. 
These results were consistent with normochromic normocytic anemia with mild polychromasia. There was also marked thrombocytopenia and microangiopathic anemia on the film, features suggestive of relapse of known HUS. Biochemistry results showed a lactate dehydrogenase (LDH) level of 1,201 U/L, haptoglobin $<0.06 \mathrm{~g} / \mathrm{L}$, potassium $3.7 \mathrm{mmol} / \mathrm{L}$, urea $20.7 \mathrm{mmol} / \mathrm{L}$, creatinine $202 \mathrm{umol} / \mathrm{L}$, estimated glomerular filtration rate $27 \mathrm{~mL} /$ minute, and C-reactive protein (CRP) of $27 \mathrm{mg} / \mathrm{L}$. High levels of LDH and low haptoglobin levels pointed to severe hemolysis while the renal markers, such as creatinine, urea, and estimated glomerular filtration rate showed a deteriorating renal function. Earlier attempts to perform the biochemical tests had been futile due to severe hemolysis.

After a multidisciplinary team collaboration that involved the Intensive Care Unit, Hematology, Nephrology, and the Emergency Department, a left femoral vascath was inserted with the view of commencing TPE using $3 \mathrm{~L}$ of fresh frozen plasma (FFP). The procedure was scheduled to be done in the emergency department using a membrane based TPE machine (mTPE). Plasma FLUX PSu 2S plasma exchange filters manufactured by Fresenius SE \& Co (Bad Homberg, Germany) were used. These filters have a surface area of $0.6 \mathrm{~m}^{2}$, blood priming volume of $70 \mathrm{~mL}$, and a plasma sulphone membrane. Filtration is primarily based on pressure gradients allowing filtration of molecules of up to $1,000 \mathrm{kDa}$ including immunoglobulins, complement factors, and albumin. Soon after commencing TPE, the machine showed a blood leak alarm and it was evident that the membrane had ruptured gauging by the color of the effluent (Figure 1). The filter was replaced with another one which also did not last long before the machine showed a blood leak alarm. The procedure was aborted after exchanging only three bags of FFP. An attempt was made to resume treatment with a centrifuge based TPE machine (cTPE). The centrifugal device we used was a Spectra Optia Apheresis System, a product of Terumo BCT (Lakewood, CO, USA). This machine operates by separating blood products according to their specific gravity using centrifugal force. The spill over alarm persisted on cTPE and "red blood cell detected" was shown on the machine. At this point, it was agreed to stop TPE due to the nature of the technical problems which were attributed to severe hemolysis.

On day 2, it was agreed that the patient could be treated with eculizumab (complement C5 blocker), but the drug was not available for compassionate access. We then revisited cTPE and the consultant overseeing our cTPE machine

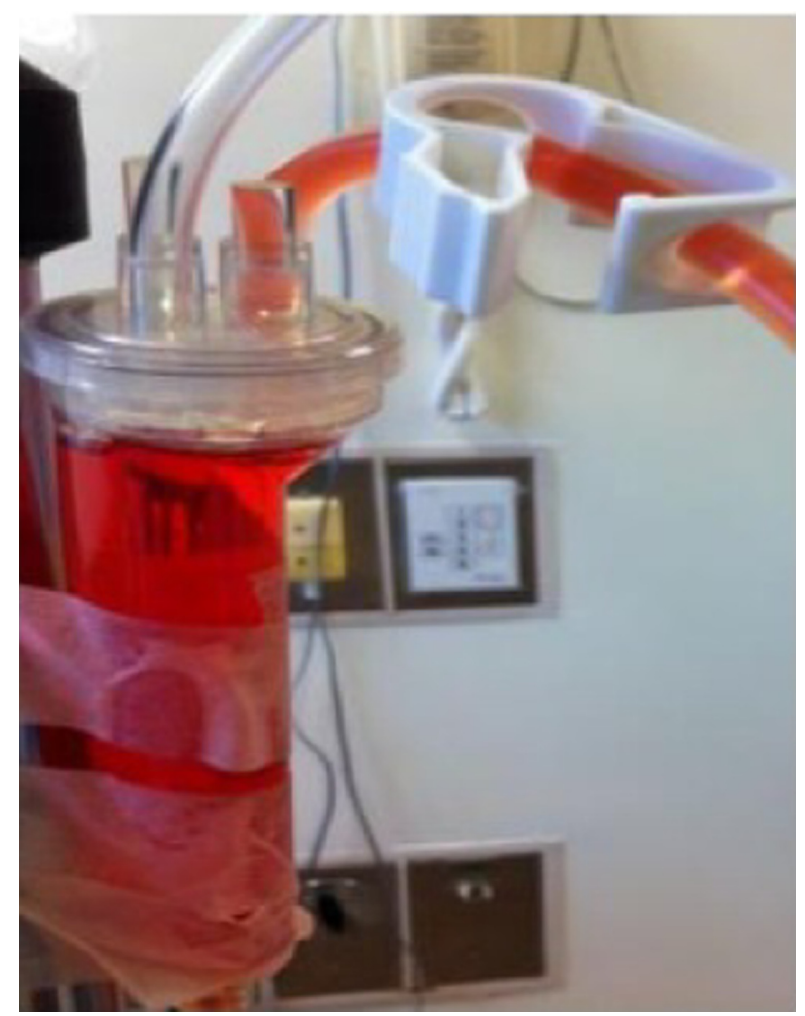

Figure I mTPE effluent after blood leak.

Abbreviation: mTPE, membrane based therapeutic plasma exchange.

was contacted. We were advised to "disable" the red blood cell detector and cTPE was initiated successfully using a very low inlet flow rate ranging from $20-70 \mathrm{~mL} /$ minute with an anticoagulant infusion rate of $1.0 \mathrm{~mL} /$ minute. Heavily hemolyzed effluent was noted (Figure 2). The patient continued to receive daily cTPE until day 16 when a decision was made that she had reached clinical remission, and she was discharged home. Her hematological (Table 1) and biochemical (Figure 3) results continued to improve. A follow up of this patient after 6 weeks revealed that she was clinically well and had resumed her normal daily routines. However, plans had been made for her to have a permanent vascular access in the form of an arterio-venous fistula (AVF) in the setting of these recurrent aHUS episodes which seem to resolve after aggressive TPE treatment.

\section{Discussion}

Both machines (mTPE and cTPE) were fitted with safety mechanisms to prevent loss of red blood cells during treatment and if there was a blood leak, an alarm was triggered and the machine would stop automatically. Interestingly, in our case, we knew that the effluent was very rich in hemolysis products and the machine that won the day was one that 


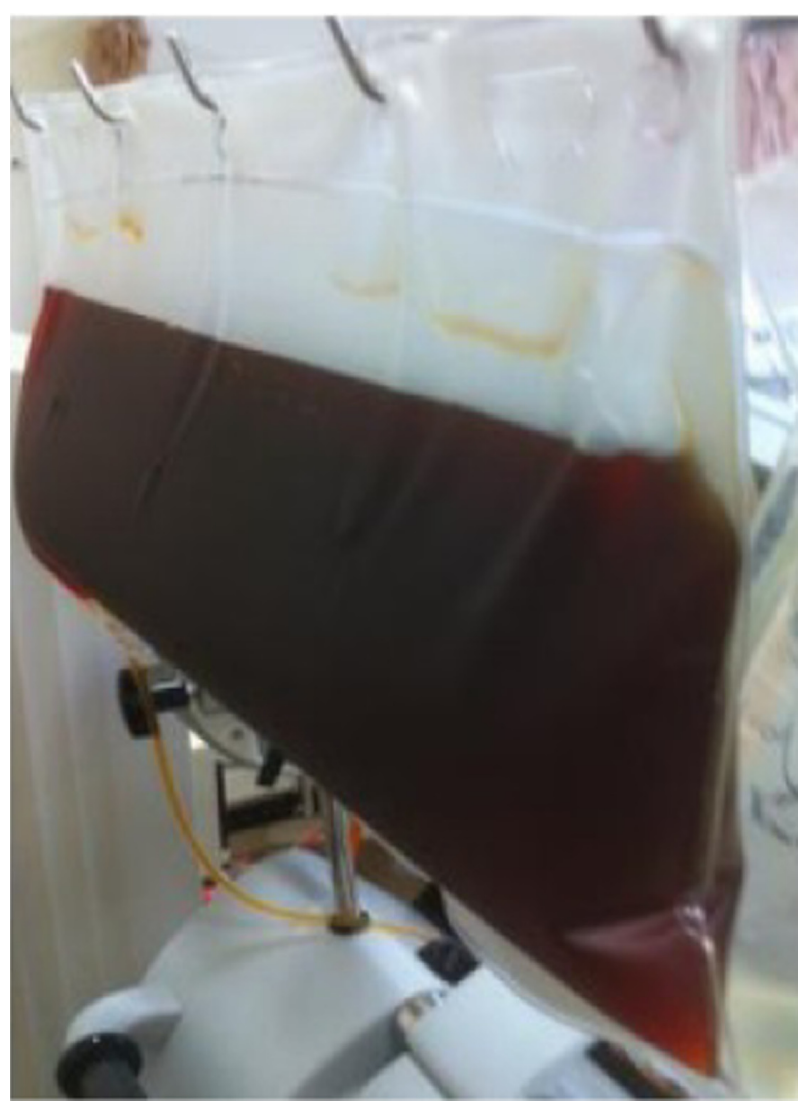

Figure 2 Heavily hemolyzed effluent on day I of cTPE.

Abbreviation: cTPE, centrifuge based therapeutic plasma exchange.

we could manipulate easily to tailor our treatment plan; this was the cTPE. Furthermore, another dilemma with using the mTPE is that plasma has different optical properties from ultrafiltrate, which can set off the blood leak alarm and stop the pump even if there is no actual blood leak. In this respect, nursing staff were not comfortable overriding the mTPE blood leak alarm since it is challenging to determine whether there is an actual blood leak or not. The cTPE also gave us an opportunity to assess the color changes of the patient's effluent and this boosted our confidence as we visually noticed some positive results of the treatment.
We believe that our patient received the best TPE treatment we could offer since some clinical cross over trials have reinforced the notion that cTPE allows a higher plasma exchange rate compared to $\mathrm{MTPE}$ without adverse effects on treatment quality and tolerability. ${ }^{4}$ This sentiment has been shared by another researcher who has found that plasma removal is more efficient with cTPE compared to mTPE systems, which have a lower plasma extraction ratio and therefore require longer procedure times. ${ }^{5}$ The cTPE treatment also ensured that our patient received the $3 \mathrm{~L}$ of FFP that was ordered and that an equal volume of patient plasma was removed. From our experience with dialysis, in patients whose post dialysis weight does not correlate with the reported ultrafiltration, the preciseness of the built-in ultrafiltration controller in the MTPE machine is questionable.

FFP was used as a substitution solution during TPE despite the high rate of side effects, such as anaphylactic reactions ${ }^{6,7}$ that may actually require cessation of plasmatherapy. ${ }^{8} \mathrm{We}$ used FFP because it provides normal amounts of complement factors (CFH, CFI, CFB, and $\mathrm{C} 3$ ) and functional proteins ${ }^{9}$ which are vital for treating aHUS.

It was unfortunate that our patient could not access eculizumab, which is an anti-complement factor 5 (C5) monoclonal antibody that binds to $\mathrm{C} 5$ thereby preventing activation of the terminal complement cascade. Recent impressive improvement in the management of aHUS has been reported with the use of eculizumab which is becoming the new breakthrough treatment option for patients with primary aHUS, providing improved control of the disease over plasma exchange, with a good safety profile.$^{10}$ However, it is still not quite clear whether life-long or recurrence-specific treatment is necessary and how genetics may or may not impact care of persons on eculizumab. ${ }^{11}$ What is known, however, is that defects in more than one complement regulator in aHUS cases may pose significant therapeutic challenges. ${ }^{12}$ Recent reports indicate that mutations in $D G K E$ (which encodes diacylglycerol kinase $\varepsilon$ ) were

Table I Hematological parameters

\begin{tabular}{|c|c|c|c|c|c|c|c|c|c|c|}
\hline $\begin{array}{l}\text { Hematological } \\
\text { parameters }\end{array}$ & D I & D 2 & D 3 & D 4 & D 5 & D 8 & D 9 & D IO & D 15 & Wk 6 \\
\hline Hemoglobin (g/L) & 105 & 73 & 73 & 71 & 74 & 78 & 72 & 76 & 81 & 127 \\
\hline Platelets $\left(\times 10^{9} / \mathrm{L}\right)$ & 16 & 15 & 21 & 13 & 12 & 87 & 105 & $12 \mid$ & 262 & 163 \\
\hline Hematocrit (\%) & 30 & 20 & 20 & 19 & 20 & 22 & 21 & 22 & 23 & 37 \\
\hline $\begin{array}{l}\text { Red cell count } \\
\left(\times 10^{17} / \mathrm{L}\right)\end{array}$ & 3.36 & 2.3 & 2.3 & 2.22 & 2.26 & 2.42 & 2.27 & 2.34 & 2.43 & 3.99 \\
\hline
\end{tabular}

Note: Data shown are for selected days only when the patient was in hospital and 6 weeks after discharge.

Abbreviation: D, day. 


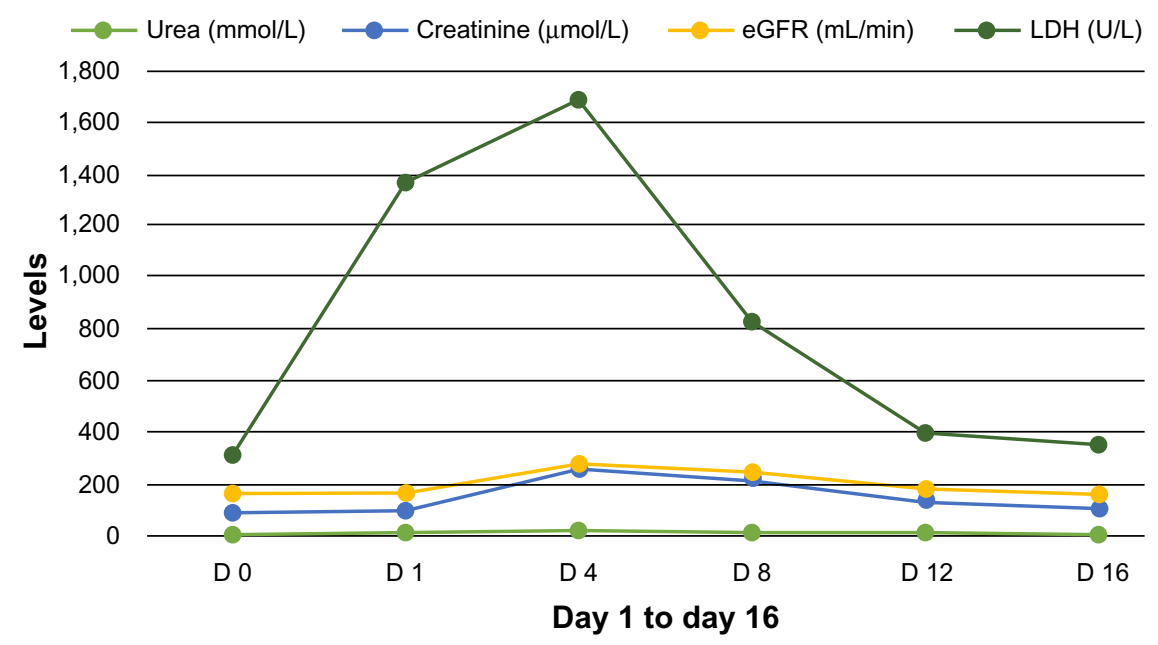

Figure 3 Biochemical parameters.

Abbreviations: D, day; eGFR, estimated glomerular filtration rate; LDH, lactate dehydrogenase.

found in several aHUS patients and this may have an impact on the successful treatment of individuals with eculizumab. ${ }^{13}$ Nevertheless, we are delighted that we successfully delivered first line treatment ${ }^{8}$ for aHUS through cTPE.

\section{Conclusion}

Traditionally, cTPE is the method preferred by hematology or blood bank based physicians for plasma exchange while, on the other hand, nephrology based physicians prefer mTPE. From our experience, it may be better to individualize these treatment options since patient needs vary. For a patient with aHUS, treating with centrifuge devices such as the Spectra Optia Apheresis System (Terumo BCT) may result in better outcomes. Clinicians also seem to be more comfortable managing the technical challenges, such as the continuous activation of blood leak alarms caused by severe hemolysis for patients on cTPE compared to MTPE.

\section{Disclosure}

The author reports no conflicts of interest in this work.

\section{References}

1. Noris M, Remuzzi G. Atypical hemolytic-uremic syndrome. $N$ Engl J Med. 2009;361(17):1676-1687.
2. Constantinescu AR, Bitzan M, Weiss LS, et al. Non-enteropathic hemolytic uremic syndrome: causes and short-term course. Am J Kidney Dis. 2004;43(6):976-982.

3. Kavanagh D, Goodship TH. Atypical hemolytic uremic syndrome, genetic basis, and clinical manifestations. Hematology Am Soc Hematol Educ Program. 2011;2011:15-20.

4. Golla P, Hafer C, Clajus C, Schmidt BM, Kielstein J. Membrane versus Centrifuge based therapeutic plasma exchange-a clinical-cross over comparison. NDT-Plus. [Abstract] 2011;4(2):S2.37.

5. Ward DM. Conventional apheresis therapies: a review. J Clin Apher. 2011;26(5):230-238

6. Asif N, Hassan K. Fresh frozen plasma. Hematology updates. 2011: 108-111.

7. Campistol JM, Arias M, Ariceta G, et al. An update for atypical haemolytic uraemic syndrome: diagnosis and treatment. A consensus document. Nefrologia. 2013;33(1):27-45.

8. Loirat C, Garnier A, Sellier-Leclerc AL, Kwon T. Plasmatherapy in atypical hemolytic uremic syndrome. Semin Thromb Hemost. 2010;36(6):673-681.

9. Loirat C, Fremeaux-Bacchi V. Atypical hemolytic uremic syndrome. Orphanet J Rare Dis. 2011;6(1):60.

10. Zuber J, Fakhouri F, Roumenina LT, Loirat C, Frémeaux-Bacchi V; French Study Group for aHUS/C3G. Use of eculizumab for atypical haemolytic uraemic syndrome and C3 glomerulopathies. Nat Rev Nephrol. 2012;8(11):643-657.

11. Bu F, Borsa N, Gianluigi A, Smith RJ. Familial atypical hemolytic uremic syndrome: a review of its genetic and clinical aspects. Clin Dev Immunol. 2012;2012:370426.

12. Waters AM, Licht C. aHUS caused by complement dysregulation: new therapies on the horizon. Pediatr Nephrol. 2011;26(1):41-57.

13. Lemaire M, Frémeaux-Bacchi V, Schaefer F, et al. Recessive mutations in DGKE cause atypical hemolytic-uremic syndrome. Nat Genet. 2013;45(5):531-536.

\section{Publish your work in this journal}

The International Journal of Nephrology and Renovascular Disease is an international, peer-reviewed open-access journal focusing on the pathophysiology of the kidney and vascular supply. Epidemiology, screening, diagnosis, and treatment interventions are covered as well as basic science, biochemical and immunological studies. The journal welcomes original research, clinical studies, reviews \& evaluations, expert opinion and commentary, case reports and extended reports. The manuscript management system is completely online and includes a very quick and fair peerreview system, which is all easy to use. Visit http://www.dovepress.com/ testimonials.php to read real quotes from published authors. 